\title{
Anomalous Mass Number Dependence of Nuclear Transparency
}

\author{
Akihisa KoHAMA, Koichi YAZAKI and Ryoichi SEKI* \\ Department of Physics, University of Tokyo, Tokyo 113, Japan \\ *Department of Physics and Astronomy \\ California State University, Northridge, CA 91330 and \\ W. K. Kellogg Radiation Laboratory \\ California Institute of Technology, Pasadena, CA 91125, U. S. A.
}

\begin{abstract}
We examine target mass number $(A)$ dependence of the nuclear transparency calculated for $\left(e, e^{\prime} p\right)$ reactions. Generally, the transparency is proportional to $A^{-1 / 3}$ for large $A$ 's and has weaker $A$-dependence as $A$ decreases. The conventional nuclear physics calculation yields the transparency whose $A^{-1 / 3}$ dependence is reached in rather light nuclei (around $A$ $=20$ ). If either the proton mean-free path in nuclei becomes substantially longer, or the internal structure of the proton is explicitly incorporated in the calculation, however, the $A^{-1 / 3}$ dependence is reached only in extremely heavy nuclei. The $A$-dependence should thus serve as a signature of the so-called color-transparency phenomenon.
\end{abstract}

\section{§ 1. Introduction and conclusions}

Nuclear (color) transparency reflects internal dynamics of hadrons, as was proposed by Brodsky and Mueller on the basis of perturbative Quantum Chromodynamics (QCD). ${ }^{1} \quad$ The phenomenon of nuclear transparency, however, has been found to be more complicated than was originally proposed, and its clear signature is difficult to obtain.

Experimental results on the nuclear transparency in $\left(e, e^{\prime} p\right)$ have been reported recently. ${ }^{2,3)}$ The results of Ref. 2) indicate a weak energy dependence and a slow onset of the nuclear transparency.

In the previous publications, ${ }^{4)}$ we calculated the nuclear transparency in the conventional multiple-scattering theory. Our approximations employed in these papers are valid only for a relatively high momentum transfer, $|\boldsymbol{q}|$, and the numerical result presented is for the high $|\boldsymbol{q}|$ region, $|\boldsymbol{q}| \geq 2[\mathrm{GeV} / c]$, where the proton-nucleon reaction and total cross sections are practically independent of the incident momentum. Our transparency prediction was 0.68 for ${ }^{12} \mathrm{C}\left(e, e^{\prime} p\right)$, which agrees with the recent observation ${ }^{2)}$ within the experimental uncertainties for $|\boldsymbol{q}| \geq 2[\mathrm{GeV} / c]$. Note, however, that momentum cuts were imposed in the experiment. If the experimental results would still depend on the upper limit of the missing momentum, $\boldsymbol{p}_{m}=\boldsymbol{p}^{\prime}-\boldsymbol{q}$, the resulting transparency should become larger.

In this work, we examine the atomic mass number $(A)$ dependence of nuclear transparency and propose that the $A$-dependence, rather than the magnitude of the 
transparency itself for each nucleus, should serve as a signature of the phenomenon.) If the transparency should be observed to go up as the momentum transfer increases, the observation would certainly be a clear signature of the phenomenon. Such an observation seems to be difficult to come by, and we consider the $A$-dependence as a useful means in view of the present experimental situation.

The $A$-dependence has been utilized in a phenomenological analysis of data by Jain and Ralston. ${ }^{6), 7)}$ Their objective is to extract the signature from the data as directly as possible, depending least on specific model calculations. We argue, however, that calculations currently available could serve as a reasonable means for extracting the signature when they are treated with care. The underlying expectation that we have in mind (and will demonstrate as is the case) is that the signature of the nuclear transparency is robust. We indeed find our results to be rather similar to those of Jain and Ralston in spite of their practically opposite approaches.

The proposal to use the $A$-dependence as a signature of the transparency is certainly not new, as it was made in an earlier work by Farrar et al. ${ }^{8)}$ However we now know that the phenomenon is far more complicated than was foreseen earlier.

We confine ourselves to the $\left(e, e^{\prime} p\right)$ reaction in this work, while we emphasize that our point is equally applicable to other more complicated, semi-exclusive reactions, such as $(p, 2 p)$. We will make detailed comparison of more elaborate calculations, (1) a nuclear-physics calculation based on a multiple-scattering theory, ${ }^{4)}$ and (2) a QCD-motivated calculation using a dynamical model associated with the internal structure of the proton. ${ }^{9)}$ These are previous works of our own, and as such, there arises a natural question of generality in our conclusion drawn from their close comparison.

As pointed out by Jain and Ralston, ${ }^{6)}$ one should be extremely careful to choose the quantity that would serve as a signature of the nuclear transparency. As described with great care in our previous work, ${ }^{4)}$ we define the nuclear transparency, $T(\boldsymbol{q})$, as a function of the momentum transfer, $\boldsymbol{q}$, as

$$
T(\boldsymbol{q})=\frac{1}{Z} \frac{1}{A(\boldsymbol{q})} \frac{d \sigma_{e A}}{d \Omega_{k^{\prime}}} / \frac{d \sigma_{e p}}{d \Omega_{k^{\prime}}},
$$

where $Z$ is the atomic number of the target nucleus, and $A(\boldsymbol{q}) \simeq 1.05$ is the Fermimotion averaging factor, almost independent of $\boldsymbol{q}=\boldsymbol{k}-\boldsymbol{k}^{\prime}{ }^{4)}$ Here $\boldsymbol{k}$ is a momentum of the incident electron, and $\boldsymbol{k}^{\prime}$ is that of the outgoing electron. Note that the quasi-elastic cross sections in Eq. (1.1) are results of integration over the struckproton momentum, $\boldsymbol{p}^{\prime}$, and over the magnitude of the momentum of the outgoing electron, $\boldsymbol{k}^{\prime}$.

We refer the reader to Ref. 4) for detailed discussions leading to the preceding definition of the nuclear transparency as well as the kinematical considerations involved. Note that we use the relativistic kinematics for the electrons and the struck proton throughout.

\section{§ 2. Formulation}

We examine $T(\boldsymbol{q})$ obtained by two calculations: (1) the Glauber Impulse Approxi- 
mation (GIA), ${ }^{4)}$ which is based on the Glauber multiple-scattering (not eikonal) theory, and (2) the $b$-model, ${ }^{9)}$ which incorporates the dynamical structure of the proton. We call the proton dynamical (inert) when its internal structure is (not) taken into account. In the following, we list the key expressions used in this work. Their detailed derivations are given in the references cited.

(1) The GIA is applied under zero-range-no-recoil (ZRNR) approximation, which neglects the target nuclear recoil, and the finite $N N$-interaction range in the final-state interaction. We have

$$
T(\boldsymbol{q})=\int d \boldsymbol{r} \rho(\boldsymbol{r}) P^{(-)}(\boldsymbol{r}),
$$

where without the nuclear correlation,

$$
P^{(-)}(\boldsymbol{r})=\exp \left\{-(A-1) \sigma_{N N}^{r} \int_{\zeta}^{\infty} d \zeta^{\prime} \rho\left(\boldsymbol{b}, \zeta^{\prime}\right)\right\} .
$$

Here, $\boldsymbol{r}=(\boldsymbol{b}, \zeta), \rho(\boldsymbol{r})$ is the nuclear density, normalized to unity, and the path of the integral in Eq. $(2 \cdot 2)$ is taken to be along the classical path of the struck proton. $\sigma_{N N}^{r}$ is the proton-nucleon reaction cross section, $\sigma_{N N}^{r}=\sigma_{N N}^{\text {total }}-\sigma_{N N}^{\text {elastic }}$. For the numerical results shown below, we use $\sigma_{N N}^{r}=28[\mathrm{mb}]$ for the struck proton momentum of about $2-15[\mathrm{GeV} / c]$ where the reaction cross section is practically constant.

In our previous works, ${ }^{4)}$ we proposed to use the mean-free path determined by the proton-nucleon reaction cross section, not by the total cross section in this energy region. The use of the reaction cross section is justified only if the experiment is "inclusive" with respect to the residual nucleus. We consider that the experiment of Ref. 2) is inclusive enough in this sense, because they integrated over the missing energy, $E_{m}=\nu-T^{\prime}-T_{B}$, where $T_{B}$ is a kinetic energy of the residual $(A-1)$-body nucleon system, and they showed the transparency to have little dependence on the upper bound.

(2) For the $b$-model describing the dynamical proton, we have

$$
P^{(-)}(\boldsymbol{r})=\frac{1}{\left|F_{e p}\left(q^{2}\right)\right|^{2}}\left|M_{e A}^{(D)}\left(q^{2}, t(\boldsymbol{r})\right)\right|^{2},
$$

where the survival amplitude for the dynamical proton is defined by

$$
M_{e A}^{(D)}\left(q^{2}, t\right)=\left\langle\varphi_{0,0}\left|e^{-i H t} e^{-b^{2} q^{2} / 6}\right| \varphi_{0,0}\right\rangle .
$$

Here, our initial condition at $t=0$, when the proton is hit by a photon at $r$, is that the proton wave function is $e^{-b^{2} q^{2} / 6}\left|\varphi_{0,0}\right\rangle$, where $\left|\varphi_{0,0}\right\rangle$ is the lowest eigenfunction of $H_{0}$ of Eq. $(2 \cdot 5)$ below, and $t(r)$ is the time spent by the proton to get out of the nucleus. This means that the small- $b$ components are favored when $|\boldsymbol{q}|$ is large. $H$ in Eq. $(2 \cdot 4)$ is the hamiltonian of the $b$-model, consisting of the proton internal hamiltonian, $H_{0}$, and its interaction with the nuclear medium, $H_{1}$, i.e., ${ }^{10)}$

$$
H_{0}=-\frac{1}{2 m_{b}}\left(\frac{d^{2}}{d b^{2}}+\frac{3}{b} \frac{d}{d b}\right)+\frac{1}{2} k_{b} b^{2}, \quad H_{1}=-i \theta(t) \alpha(\beta b)^{2},
$$

where $m_{b}$ and $k_{b}$ are parameters defined as $k_{b}=\beta^{2} \omega_{b}$, and $m_{b}=\beta^{2} / \omega_{b} . \quad \beta=1.66\left[\mathrm{fm}^{-1}\right]$ 
and $\omega_{b}=250[\mathrm{MeV}]$. ( $\alpha$ here is $c_{0}$ in Ref. 9).) $\alpha$ expresses a strength of absorption, which is determined by $4 \alpha=\lambda^{-1}=94[\mathrm{MeV}]$. Here, $\lambda=2.1[\mathrm{fm}]$ is the mean-free path of the proton by the use of $\sigma_{N N}^{r}$. Note that we recover the amplitude for the inert proton by replacing $H_{1}$ with its ground state expectation value.

The effect of smooth nuclear surface is approximately included by taking $t(\boldsymbol{r})$ as

$$
t(\boldsymbol{r})=\frac{1}{v_{q} \rho_{00}} \int_{\zeta}^{\infty} d \zeta^{\prime} \rho\left(\boldsymbol{b}, \zeta^{\prime}\right),
$$

where $v_{q}$ is the velocity of the struck proton, and $\rho_{00}$ is the value of $\rho(\boldsymbol{r})$ at the origin. Equation $(2 \cdot 6)$ is valid for the weak interaction strength when the modification of the time evolution is proportional to the density. Note that the time dependence is converted to the $\boldsymbol{r}$-dependence through Eq. $(2 \cdot 6)$.

The Lorentz factor for the internal time is taken into account by replacing $\omega_{b}$ with $\sqrt{1-v_{q}^{2}} \omega_{b}$ where $v_{q}$ is the velocity of the proton with the transferred momentum, $\boldsymbol{q}$. This factor also gives rise to the $\boldsymbol{q}$-dependence of the nuclear transparency enhancing it at large $|\boldsymbol{q}|$.

\section{§3. Numerical results and discussions}

In order to discuss the $A$-dependence of the transparency, we take the WoodsSaxon density

$$
\rho(\boldsymbol{r})=\rho_{0} \frac{1}{1+e^{(r-c) / z}}, \quad \int d \boldsymbol{r} \rho(\boldsymbol{r})=1, \quad c=1.1 \times A^{1 / 3}[\mathrm{fm}], \quad z=0.53[\mathrm{fm}] .
$$

Figure 1 illustrates the transparency by the GIA without the nuclear correlation

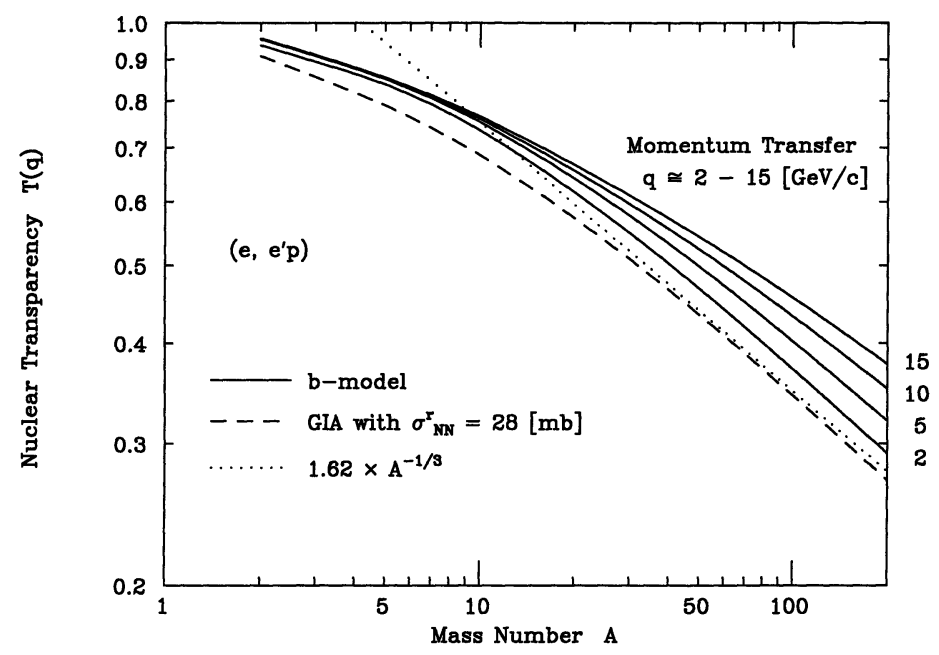

Fig. 1. Comparison of the GIA and the $b$-model in regard to the $A$-dependence of the nuclear transparency. The solid curve is the transparency by the $b$-model shown for the momentum transfer, $|\boldsymbol{q}|=2,5,10$, and $15[\mathrm{GeV} / \mathrm{c}]$ from the lowest curve to the highest. The dashed curve is that by the GIA with $\sigma_{N N}^{r}=28[\mathrm{mb}]$. The dotted line represents the asymptotic $A$-dependence of the GIA. 
and that by the $b$-model. At a relatively low-momentum transfer, $|\boldsymbol{q}|$ of $5[\mathrm{GeV} / c]$, we see that in the GIA, the $A^{-1 / 3}$ dependence is already reached at $A \simeq 20$. The dotted line corresponds to the asymptote of the transparency in the GIA with the fitted coefficient 1.62. At this momentum transfer, the slope of the transparency by the $b$-model is different only slightly from that by the GIA. When we include the nuclear correlation in the GIA, we also obtain the $A^{-1 / 3}$ dependence for $A \geq 20$, but with larger magnitude. ${ }^{5)} \quad$ Figure 1 also shows the $\boldsymbol{q}$-dependence of the transparency, and clarifies the difference between the GIA and the $b$-model. Since $\sigma_{N N}^{r}$ practically remains the same above a few $\mathrm{GeV} / c$, so does the transparency by the GIA. We thus show one curve for the GIA in Fig. 1. The transparency by the $b$-model, however, changes: Its $A$-dependence becomes weaker than $A^{-1 / 3}$, even for large $A$, as $\boldsymbol{q}$ increases. This difference should serve as a signature of the onset of dynamical effects associated with the internal structure of the proton. This is our major result in this work.

In a report on the NE18 $\left(e, e^{\prime} p\right)$ SLAC experiment, ${ }^{3)}$ they discuss observation of the $A$-dependence of nuclear transparency. In Ref. 3 ) they fit the data with a simple parameterization, $A^{\alpha}$, and obtain $\alpha<1 / 3$ with more weight on the data for light nuclei. We note that although these $\alpha$ values are different from the asymptotic $\alpha$ value $1 / 3$ that we have discussed here, there is no contradiction between this work and Ref. 3).

We now investigate the possibility that our $b$-model result in Fig. 1 can be reproduced by the GIA results with an effectively longer mean-free path or an effectively smaller $N N$ cross section. We find that this is indeed the case for a finite region of $A$ : Figure 2 shows that the GIA with $\sigma_{N N}^{\text {eff }}$ instead of $\sigma_{N N}^{r}$ yields the transparency similar to that by the $b$-model. We take $\sigma_{N N}^{\text {eff }}=22.4$, and $20.4[\mathrm{mb}]$ at $|\boldsymbol{q}|=5$, and $10[\mathrm{GeV} / c]$ respectively. This gives the effective mean-free path, $\lambda_{\text {eff }}=2.63[\mathrm{fm}]$ for $|\boldsymbol{q}|$ $=5[\mathrm{GeV} / c]$, and $\lambda_{\text {eff }}=2.88[\mathrm{fm}]$ for $|\boldsymbol{q}|=10[\mathrm{GeV} / c]$, both of which are longer than the $\lambda$ used in the original GIA. The salient point is indeed that the effective mean-free path

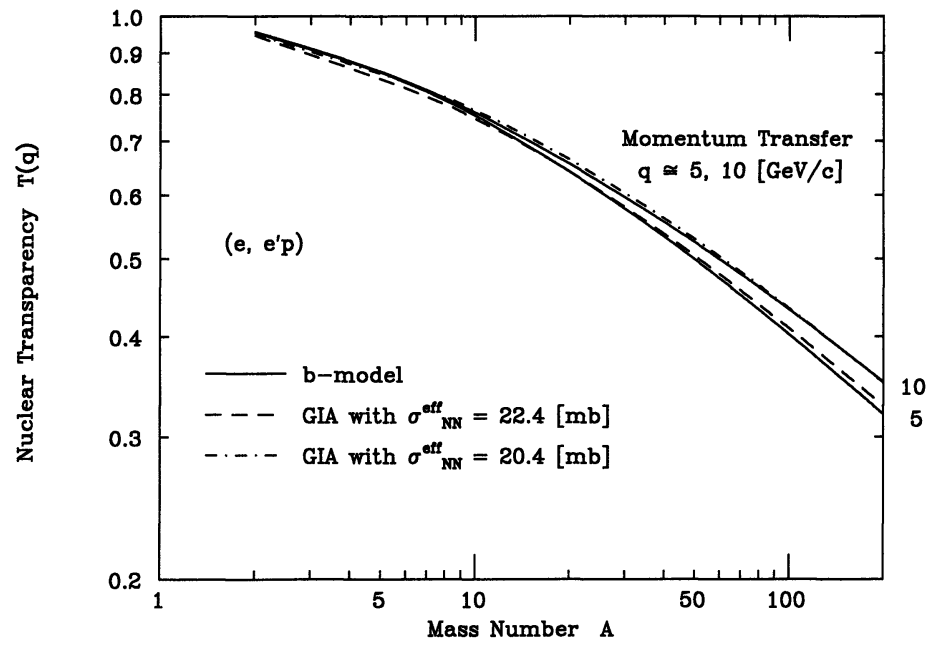

Fig. 2. Comparison of the $b$-model and the GIA with $\sigma_{N N}^{\text {eff }}$. The solid curves are the transparency by the $b$-model with the momentum transfer, $|\boldsymbol{q}|=5$ and $10[\mathrm{GeV} / c]$. The dashed curve is the transparency by the GIA with $\sigma_{N N}^{\text {eff }}=22.4[\mathrm{mb}]$, and the dash-dotted curve is the transparency by the GIA with $\sigma_{N N}^{\text {eff }}=20.4[\mathrm{mb}]$. 
in the $b$-model is thus longer than that in the conventional multiple-scattering theory. We note that the same observation regarding the smaller effective $N N$ cross section was also made by Jain and Ralston ${ }^{6), 7)}$ in spite of their approach being practically opposite to ours. They extracted phenomenologically from the $(p, 2 p)$ data, ${ }^{11)}$ i.e., $\sigma_{N N}^{\text {eff }}$ $=17[\mathrm{mb}]$ at $|\boldsymbol{q}|=2.2[\mathrm{GeV} / c]$ and $12[\mathrm{mb}]$ at $|\boldsymbol{q}|=2.9[\mathrm{GeV} / c]$.

\section{Acknowledgements}

A. K. is supported by Fellowships of the Japan Society for the Promotion of Science (JSPS) for Japanese Junior Scientists.

\section{References}

1) S. J. Brodsky, Proceedings of 13th International Symposium on Multiparticle Dynamics 1982, ed. W. Kittel, W. Metzger and A. Stergiou (World Scientific, 1982), p. 963.

A. H. Mueller, Proceedings of the 17th Rencontre de Moriond (Les Arcs, 1982), ed. J. Tran Thanh Van (Ed. Frontiers, 1982).

2) N. C. R. Makins et al., Phys. Rev. Lett. 72 (1994), 1986.

3) T. G. O'Neill et al., Preprint OAP-731, submitted to Phys. Rev. Lett.

4) A. Kohama, K. Yazaki and R. Seki, Nucl. Phys. A536 (1992), 716; A551 (1993), 687.

5) A. Kohama, K. Yazaki and R. Seki, Phys. Lett. B344 (1995), 61.

6) P. Jain and J. P. Ralston, Phys. Rev. D48 (1993), 1104.

7) J. P. Ralston, in Perspectives in Hadron Structure, ed. M. Harakeh et al. (NATO Adv. Study. Inst., Dronton, the Netherlands, 1993).

8) G. R. Farrar, H. Liu, L. L. Frankfurt and M. I. Strikman, Phys. Rev. Lett. 61 (1988), 686.

9) A. Kohama and K. Yazaki, Nucl. Phys. A575 (1994), 645.

K. Yazaki and A. Kohama, Proceedings of International School-Seminar '93 (ISS93), Hadrons and Nuclei from QCD, Tsuruga-Vladiwostok-Sapporo, ed. K. Fujii, Y. Akaishi and B. L. Reznik (World Scientific, Singapore, 1994), p. 10.

10) J. F. Gunion and D. E. Soper, Phys. Rev. D15 (1977), 2617.

11) A. S. Carroll et al., Phys. Rev. Lett. 61 (1988), 1698. 